\begin{tabular}{lll} 
Abstract 65 Table 1 & March 2016 & September 2016 \\
\hline & 135 & 120 \\
\hline No. of patients admitted with chest pain & 120 & 104 \\
Troponin assessed & 5 & 5 \\
At least one positive Troponin (>40 ng/L) & 42 & 0 \\
Admitted overnight to: & 42 & 12 \\
- assess 12 hour troponin & & \\
- for further assessment & 42 & 12 \\
Subsequent discharge after post-take ward round & 4 & 1 \\
Inpatient angiography & 9 & 5 \\
Outpatient Rapid Access Chest Pain Clinic referral & 6 & 13 \\
Readmission within 30 days with chest pain & 6 & 0 \\
Readmission within 30 days with NSTEMI & 1 & 7 \\
Mean length of stay (hours) & 20 & \\
\hline
\end{tabular}

the post-take ward round. The 12 hour troponin was never raised if the admitting troponin was negative. In September, 120 patients were assessed for chest pain; 5 had positive troponin, of which 1 was an NSTEMI. No patients were admitted overnight to wait for a 12 hour troponin result. Twelve patients were admitted overnight, despite a negative 3 hour troponin, for further assessment. All were discharged home at the post-take ward round. 6 patients were re-admitted within 30 days of discharge from the March cohort, with one fatality secondary to congestive cardiac failure. In September, there were 13 re-admissions with chest pain, none had a positive Troponin result or missed myocardial infarction. There was no change in rates of referral to the Rapid Access Chest Pain Clinic on discharge ( 9 in March, 5 in September). Based on a cost of $£ 300$ per bed day, changing to the updated pathway generated a cost saving of between $£ 100000$ to $£ 150000$ per year to the trust. Average length of stay for those admitted for the assessment of chest pain, excluding those diagnosed with other medical conditions was 20 hours in March and 7 hours following introduction of the new protocol.

Conclusion Implementation of a 3 hour rule-out troponin protocol improved the quality of care delivered to patients at MCHT. Patients were given a diagnosis more quickly and the practise of admitting patients overnight for a 12 hour troponin has stopped. This has had significant cost and bed savings for a busy district general hospital.

\section{COGNITIVE IMPAIRMENT IS NOT ASSOCIATED WITH 30 DAY MAJOR ADVERSE CARDIOVASCULAR EVENTS IN OLDER (75 YEARS) PATIENTS PRESENTING WITH NON- ST ELEVATION ACUTE CORONARY SYNDROME: AN EVALUATION FROM THE ICON1 STUDY}

${ }^{1}$ Sophie Zhaotao Gu* ${ }^{1}$ Jonathan Batty, ${ }^{2}$ Hannah Sinclair, ${ }^{2}$ Murugapathy Verrasamy, ${ }^{3}$ Alan Bagnall, ${ }^{3}$ lan Purcell, ${ }^{3}$ Richard Edwards, ${ }^{3}$ loakim Spyridopoulos, ${ }^{3}$ Mohaned Egred, ${ }^{3}$ Azfar Zaman, ${ }^{3}$ Javed Ahmed, ${ }^{1}$ Vijay Kunadian. 'Institute of Cellular Medicine, Faculty of Medical Sciences, Newcastle University; ${ }^{2}$ Institute of Cellular Medicine, Newcastle University, and Cardiothoracic Centre, Freeman Hospital; ${ }^{3}$ Cardiothoracic Centre, Freeman Hospital

\subsection{6/heartjnl-2017-311726.65}

Introduction Dementia is leading cause of death in the UK and shares many common risk factors with coronary artery disease. The relationship between cognition and major adverse cardiovascular events (MACE) in older patients presenting with non ST elevation Acute Coronary syndrome (NSTEACS) is not known.

Aim To investigate the association of cognitive impairment with 30 day MACE (mortality, hospital readmission with ACS, unplanned revascularisation, stroke and major bleeding events) in older patients presenting with NSTEACS in the ICON1 study.

Method Over a period of 34 months 277 patients 75 years of age, admitted for invasive management of NSTEACS, were enrolled into a multicentre prospective observational study. Cognitive assessment was performed by Montreal Cognitive Assessment (MoCA) test, where a cut-off of 26 is used to determine cognitive impairment. Frailty was assessed using the Fried criteria, where a score of 0 is robust, 1 or 2 is pre-frail and 3 is frail.

Results 251 patients had a MoCA score calculated at presentation (mean \pm Standard deviation $[S D]=25.1 \pm 3.3$ ), nearly half of the patients $(n=122,48.6 \%)$ had cognitive impairment. The mean age was $81.2 \pm 4.0$ years (mean \pm Standard Deviation [SD]). Patients with cognitive impairment were older compared to normal cognition group (82.2 \pm 3.9 vs. $80.2 \pm 3.9$ years, $\mathrm{p}<0.001)$, and were frail $(36.1 \%$ vs. $18.6 \%, \mathrm{p}=0.002)$. Overall $19(7.6 \%)$ patients reached MACE outcome, the rate of composite adverse outcomes were $6.6 \%$ vs. $8.5 \% \quad(p=0.555)$ respectively. No patient died at 30 day in the selected cohort and no one had ST elevation myocardial infarction. There was no difference in the occurrence of non ST elevation myocardial infarction $(0.8 \%$ vs. $0.8 \%)$, unstable angina $(0.8 \%$ vs. $2.3 \%, \mathrm{p}=0.808)$, unplanned revascularisation $(1.6 \%$ vs. $1.6 \%$, $\mathrm{p}=1.0)$, stroke/Transient Ischaemic Attack $(0 \%$ vs. $0.8 \%$, $\mathrm{p}=1.0)$, and major bleeding $(4.1 \%$ vs. $3.9 \%, \mathrm{p}=1.0)$, and readmission with ACS rate $(1.6 \%$ vs. $3.1 \%, \mathrm{p}=0.684)$ between the impaired and normal cognition groups respectively.

Conclusion Cognitive impairment is common in patients over 75 years of age with NSTEACS managed invasively. Those with significant impairment are older and frail. Short-term 30 day MACE outcomes are not different between cognition groups in this selected cohort of patients.

\section{REDUCING CHEST PAIN ADMISSIONS USING A 1 HOUR HIGH-SENSITIVITY TROPONIN-T PATHWAY}

Gavin Richards*, Gautam Sen, Alison Halliday, Sadie Thomas, Emma Stevenson, Marianne Gillings, Peter Scott. Gloucestershire Royal Hospital NHSFT

\subsection{6/heartjnl-2017-311726.66}

Introduction Chest pain is the most common presenting complaint to emergency departments in the United Kingdom. Existing management pathways for suspected Acute Coronary Syndrome utilise serial cardiac troponin measurement, typically at admission and again at 6 or 12 hours, often necessitating inpatient admission. Recent work has validated the use of a high sensitivity Troponin assay to enable risk stratification using presentation and 1 hour Troponin concentrations, with a view to reducing admissions and facilitating safe, early discharge.

Methods A 1 hour troponin pathway was implemented in a District General Hospital (DGH) at Gloucestershire Royal Hospital, using a High Sensitivity Troponin-T assay (hs-cTnT, Elecsys assay, Roche Diagnostics ${ }^{\circledR}$ ). This was trialled in the Emergency Department for 56 consecutive hours. Patients with suspected ACS had a plasma hs-cTnT concentration 